\title{
Predictors and long-term prognosis of left ventricular aneurysm in patients with acute anterior myocardial infarction treated with primary percutaneous coronary intervention in the contemporary era
}

\author{
Jieyun You ${ }^{1 \#}$, Liming Gao ${ }^{1 \#}$, Yunli Shen ${ }^{1}$, Wei Guo ${ }^{1}$, Xingxu Wang ${ }^{1}$, Qing Wan ${ }^{1}$, Xiaoyan Wang ${ }^{2}$, \\ Jian Wu ${ }^{2}$, Qi Zhang ${ }^{1}$
}

${ }^{1}$ Department of Cardiovascular Medicine, Shanghai East Hospital, Tongji University School of Medicine, Shanghai, China; ${ }^{2}$ Shanghai Institute of Cardiovascular Diseases, Zhongshan Hospital and Institutes of Biomedical Sciences, Fudan University, Shanghai, China

Contributions: (I) Conception and design: J You, L Gao, J Wu, Q Zhang; (II) Administrative support: Q Zhang; (III) Provision of study materials or patients: All authors; (IV) Collection and assembly of data: J You, L Gao, J Wu; (V) Data analysis and interpretation: J You, L Gao, Q Zhang; (VI) Manuscript writing: All authors; (VII) Final approval of manuscript: All authors.

\#These authors contributed equally to this work.

Correspondence to: Qi Zhang, MD, PhD. Department of Cardiovascular Medicine, Shanghai East Hospital, Tongji University School of Medicine, Shanghai 200120, China. Email: zhangqnh@hotmail.com; Jian Wu, MD, PhD. Shanghai Institute of Cardiovascular Diseases, Zhongshan Hospital and Institutes of Biomedical Sciences, Fudan University, Shanghai 200032, China. Email: wu.jian@zs-hospital.sh.cn.

Background: Primary percutaneous coronary intervention (PCI) has been the standard reperfusion strategy for patients with acute myocardial infarction (AMI) in the contemporary era. Meanwhile, the incidence and prognosis of left ventricular aneurysm (LVA) in AMI patients remain ambiguous. The aim of the current study is to identify the predictor and long-term prognosis of LVA in patients with acute anterior myocardial infarction.

Methods: We prospectively enrolled 942 consecutive patients with acute anterior myocardial infarction who were treated by primary PCI. The baseline characteristics, procedural features, and one-year clinical outcomes were compared between the patients with and without LVA. The primary endpoint of major adverse cardiovascular and cerebrovascular events (MACCEs) was defined as a composite of cardiac death, target vessel revascularization, and ischemic stroke. Multiple logistic regression was applied to predict LVA formation and the receiver operating characteristic (ROC) curves were plotted to evaluate the accuracy of the multivariate analysis model.

Results: The general incidence of LVA was $15.92 \%$. At one-year clinical follow-up, patients in the LVA group had significantly higher incidence of MACCEs ( $15.33 \%$ vs. $6.44 \%, \mathrm{P}<0.01$ ), mainly driven by an increased incidence of cardiac death $(8.00 \%$ vs. $2.78 \%, \mathrm{P}<0.01)$, target vessel revascularization $(5.33 \%$ vs. $2.27 \%, \mathrm{P}=0.03)$, and ischemic stroke $(4.00 \%$ vs. $1.39 \%, \mathrm{P}=0.03)$. Multivariate analysis found that longer symptom-to-balloon time (S2B) [odds ratio (OR): 1.16, 95\% confidence interval (CI): 1.11-1.21, $\mathrm{P}<0.01$ ], higher initial and residual SYNTAX score (iSS, OR: 1.19, 95\% CI: 1.14-1.24, P<0.01; rSS, OR: 1.33, 95\% CI: 1.22-1.45, $\mathrm{P}<0.01$ ), lower left ventricular ejection fraction (LVEF) (OR: 1.15, 95\% CI: 1.11-1.18, $\mathrm{P}<0.01$ ), and persistent ST segment elevation (OR: 1.89, 95\% CI: 1.06-3.38, $\mathrm{P}=0.03$ ) were independent predictors of LVA formation.

Conclusions: LVA is still common in patients with acute anterior myocardial infarction in the contemporary PCI era, and the prognosis of these patients was significantly worse during the one-year clinical follow-up. Strategies of prompt reperfusion and complete revascularization may be helpful in preventing LVA formation and improving clinical outcomes. 
Keywords: Left ventricular aneurysm (LVA); acute anterior myocardial infarction; percutaneous coronary intervention (PCI); prognosis

Submitted Nov 20, 2020. Accepted for publication Jan 29, 2021.

doi: $10.21037 /$ jtd-20-3350

View this article at: http://dx.doi.org/10.21037/jtd-20-3350

\section{Introduction}

Left ventricular aneurysm (LVA) is a disastrous complication of myocardial infarction, leading to systemic embolism, ventricular arrhythmia, heart failure, and cardiac rupture (1). In addition, LVA-related thrombi may require long-term anticoagulation therapy which increases the hemorrhagic risk (2). Thus, preventing the development of LVA is important to reduce morbidity and mortality. It was reported that rapid reperfusion and drugs preventing ventricular remodeling have reduced the incidence of LVA to the range of $5 \%$ to $15 \%$ in patients with acute myocardial infarction (AMI) (3). Previous studies in the thrombolytic era found that patients with total left anterior descending (LAD) coronary artery occlusion, proximal LAD stenosis and failure of thrombolysis were at high risk of LVA (4). Data on the determinants and outcomes of LVA are limited in patients with acute anterior myocardial infarction, who are recognized as the most high-risk subset of LVA. Although previous studies have attempted to identify some determinant factors for LVA, most studies were performed during the pre-percutaneous coronary intervention (prePCI) era without focusing on the particular population with acute anterior myocardial infarction $(5,6)$. In the current study, we sought to evaluate the predictors and long-term prognosis of LVA in acute anterior myocardial infarction patients in the contemporary PCI era. We present the following article in accordance with the STARD reporting checklist (available at http://dx.doi.org/10.21037/jtd-203350).

\section{Methods}

\section{Study design}

We prospectively enrolled 942 consecutive acute anterior myocardial infarction patients who underwent primary PCI from 2012 to 2020 in Shanghai East Hospital. LVA was diagnosed in accordance with the Coronary Artery Surgery Study (CASS) protocol (7): (I) bulge of the left ventricular wall during both diastole and systole, displaying either akinesia or dyskinesia; (II) lack of trabeculation in the involved segment; and (III) distinct demarcation of the infarcted segment. Two-dimensional transthoracic echocardiography (TTE) was performed within 3 days after admission, 1 month and 1 year during follow-up. The general incidence of LVA was $15.92 \%(n=150)$ according to TTE. The TTE measurements were performed using a Philips system (Philips CX50, Philips Healthcare, MA, USA) in accordance with the American Society of Echocardiography guidelines. Left ventricular ejection fraction (LVEF) was attained by a biplane modified Simpson's method. The 18-lead electrocardiograms (EKGs) were recorded for all patients on admission and before discharge. Permanent ST-segment elevation was confirmed as the ST-segment persistent elevated $\geq 50 \%$ of the peak ST-segment level until discharge. All data were evaluated by at least two experienced investigators who were unaware of the clinical, angiographic and outcome data. The exclusion criteria were as follows: (I) non-ischemic cardiomyopathy; (II) severe valve disease; (III) congenital heart disease; (IV) myocarditis; (V) atrial fibrillation; (VI) aortoarteritis; (VII) angiodysplasia; (VIII) previous coronary artery bypass grafting or aneurysm resection surgery; (IX) malignant tumor or expected life expectancy less than 1 year; and $(\mathrm{X})$ not surviving within 3 days after admission or lost follow-up (Figure 1).

Specially trained research staffs communicated with the patients at least once a month to confirm good compliance and obtained follow-up data at 1, 3, 6, 9 and 12 months after PCI. One-year clinical endpoints were compared between the two groups.

This study was approved by the Institutional Review Board of Shanghai East Hospital (No. 2020-096) and conducted following the principles of the Declaration of Helsinki (as revised in 2013). The written informed consent was obtained from all the patients.

\section{PCI procedure and definition}

Acute anterior myocardial infarction is defined in 


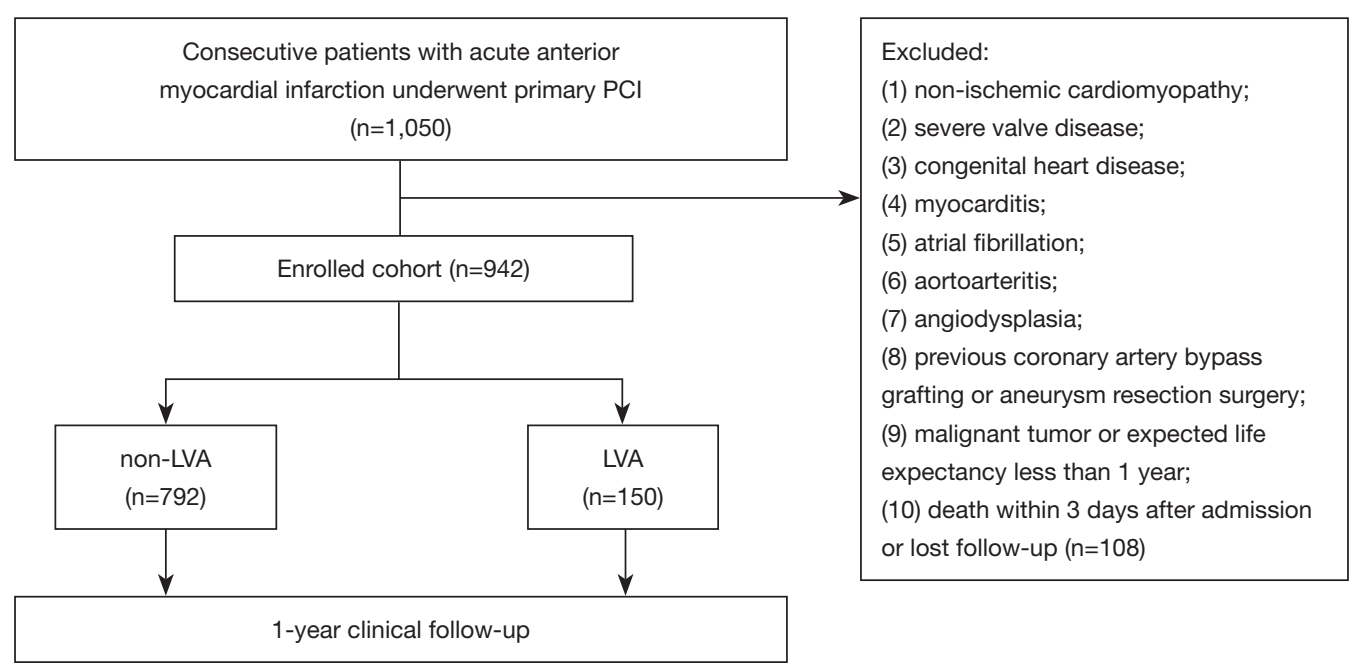

Figure 1 Study flowchart. PCI, percutaneous coronary intervention; non-LVA, patients without left ventricular aneurysm; LVA, patients with left ventricular aneurysm.

accordance with the definition of the ESC/ACCF/AHA/ WHF guidelines (8): (I) symptoms of myocardial ischemia; (II) cardiac troponin values (cTn) level above the 99th percentile upper reference limit (URL); and (III) dynamic change with ST-segment elevation or depression in the EKG compatible with acute anterior myocardial infarction. The location of myocardial infarction was reconfirmed by echocardiography and angiography.

Primary PCI was performed according to the current guideline recommendations (9-11). All patients were treated in the emergency department with DAPT consisting of aspirin (300 mg loading dose followed by $100 \mathrm{mg}$ daily), clopidogrel (of $600 \mathrm{mg}$ loading dose followed by $75 \mathrm{mg}$ daily) or ticagrelor (180 mg loading dose followed by $90 \mathrm{mg}$ twice daily). DAPT was continued for at least 12 months after PCI. Procedural anticoagulation of 70-100 U/kg unfractionated heparin was administrated at preliminary stage and supplemented on demand to maintain an activated clotting time of (ACT) >250 s. For patients with ventricular thrombus, low molecular weight heparin (LMWH) and DAPT were administered during hospitalization, and followed by triple antithrombotic therapy (aspirin $100 \mathrm{mg}$ daily, clopidogrel $75 \mathrm{mg}$ daily and warfarin) after discharge to achieve a target international normalized ratio (INR) of 1.5 to 2.0 for at least 3 months. Only the culprit lesion was intervened during primary PCI. For patients with multivessel lesions, staged PCI was planned during hospitalization or within 1 month after discharge. At discharge, medical therapy was prescribed according to the patient's status and guideline recommendations for secondary prevention.

Symptom-to-balloon time (S2B) was identified as the time interval between the chest pain onset and the first balloon inflation. The initial SYNTAX score (iSS) was analyzed according to the pre-procedural angiograms, whereas the residual SYNTAX score (rSS) was based on the post-procedural angiograms. Two experienced interventional cardiologists calculated the iSS and rSS using the online SYNTAX score I algorithm (http://www. syntaxscore.com/calculator/syntaxscore/frameset.htm). In case of disagreement, the opinion of a third analyst was obtained, and the final decision was achieved by consensus. All analysts were blinded to the baseline clinical characteristics and clinical outcomes.

\section{Endpoints}

The primary endpoint of major adverse cardiovascular and cerebrovascular events (MACCEs) was defined as a composite of cardiac death, target vessel revascularization, and ischemic stroke. The secondary endpoints included cardiogenic shock, cardiac rupture, ventricular thrombus, and major bleeding.

Cardiac death included any death without a definite noncardiac cause. Cardiogenic shock implicated a series of cardiac disorder that resulted in both clinical and biochemical evidence of tissue hypoperfusion $(12,13)$. Target vessel revascularization was recognized as ischemia referable 
to the target vessel requiring repeated PCI or bypass surgery. Ischemic stroke was considered as ischemic injury of central nervous system verified by neuropathological examination (NIHSS) and neuroimaging (computed tomography or magnetic resonance imaging) (14). Major bleeding was identified as Bleeding Academic Research Consortium (BARC) type $\geq 3$ bleeding (15).

\section{Statistical analysis}

GraphPad Prism 7.0 (GraphPad Software, CA, USA) and SPSS 25.0 (SPSS Inc., IL, USA) were used for data analyses. Demographic and clinical characteristics, medication at discharge, and PCI features were identified using univariate analysis. Continuous variables were presented as the mean with SD, while frequency counts and percentages were presented for categorical variables. The $t$-test or the Chi-square test was used to compare the continuous or nonparametric variables, respectively. Multiple logistic regression analysis was used to identify independent predictors of LVA. All factors with $\mathrm{P}<0.1$ in univariate analysis were included in multiple logistic regression analysis. Receiver operating characteristic (ROC) curves were plotted to evaluate the accuracy of the multivariate analysis model. Kaplan-Meier curves and the log-rank test were utilized to assess the prognosis of LVA. The odds ratio (OR) and the 95\% confidence interval (CI) were calculated. The sample size was determined by PASS 15 .

\section{Results}

\section{Baseline characteristics of the study population}

Total 942 patients were selected as the LVA group (15.92\%). The demographic features were similar between the non-LVA and LVA groups (all P>0.05). Impaired LVEF indicated that the LVA group was characterized with cardiac dysfunction $(\mathrm{P}<0.01)$. More patients in the LVA group manifested QS waves and persistent ST segment elevation in the electrocardiographic leads corresponding to the anterior wall $(\mathrm{P}<0.05)$. Given that the incidence of ventricular thrombus was significantly increased in the LVA group, triple anti-thrombotic therapy (DAPT + warfarin) was more administered to the LVA group than the nonLVA group $(\mathrm{P}<0.01)$. Other medications were comparable between the two groups $(\mathrm{P}>0.05)$. Longer $\mathrm{S} 2 \mathrm{~B}$ was spent in the LVA group compared with the non-LVA group $(\mathrm{P}<0.01)$. According to the angiography, the LVA group presented with significantly higher iSS and $\mathrm{rSS}(\mathrm{P}<0.01)($ Table 1).

\section{Clinical endpoints}

The composite primary endpoint of MACCEs significantly increased in the LVA group during the 1-year followup $(15.33 \%$ vs. $6.44 \%, \mathrm{P}<0.01)$, mainly driven by an increased incidence of cardiac death, target vessel revascularization, and ischemic stroke $(\mathrm{P}<0.05)$. Among the secondary endpoints, the occurrence of cardiogenic shock, cardiac rupture, and ventricular thrombus also increased considerably in the LVA group $(\mathrm{P}<0.01)$. More frequent application of triple anti-thrombotic therapy (DAPT and warfarin) increased the risk of major bleeding in the LVA group $(\mathrm{P}<0.01)$ (Table 2, Figure 2).

\section{Predictors of LVA}

Results of the multivariate logistical regression analysis indicated that for patients with acute anterior myocardial infarction who were treated by primary PCI, the independent predictors of LVA formation were longer S2B, increased iSS and rSS, impaired LVEF, and persistent ST segment elevation (Figure 3).

Based on the ROC curve analysis, the cutoff value and the area under the curve (AUC) demonstrated impaired LVEF ( $<40.50 \%)$, longer S2B ( $>3.25 \mathrm{~h})$, increased iSS $(>18.75)$ and rSS $(>3.75)$ were independently correlated with the occurrence of LVA. Combining the Syntax score and S2B together could more accurately predict LVA formation (iSS + S2B: AUC =0.85, 95\% CI, 0.82-0.88, P<0.01; rSS $+\mathrm{S} 2 \mathrm{~B}$ : AUC $=0.84,95 \% \mathrm{CI}, 0.81-0.87, \mathrm{P}<0.01$ ) (Figure 4, Table 3).

\section{Discussion}

\section{Incidence and predictors of LVA}

LVA is defined as regional akinesia or dyskinesia of the infarcted myocardium that bulges outward during both systole and diastole (7). It is a serious sequela of left ventricular remodeling caused by AMI and is related to deteriorative clinical outcomes (1). Previous studies showed that the incidences of LAD artery stenosis in LVA and nonLVA were $89.6 \%$ and $69.6 \%$, respectively (16). Given that the LAD artery provides the major blood supply for the anterior wall while not easily forming collateral circulation, it is prone to LVA formation after acute anterior myocardial 
Table 1 Baseline characteristics and treatment of the two groups

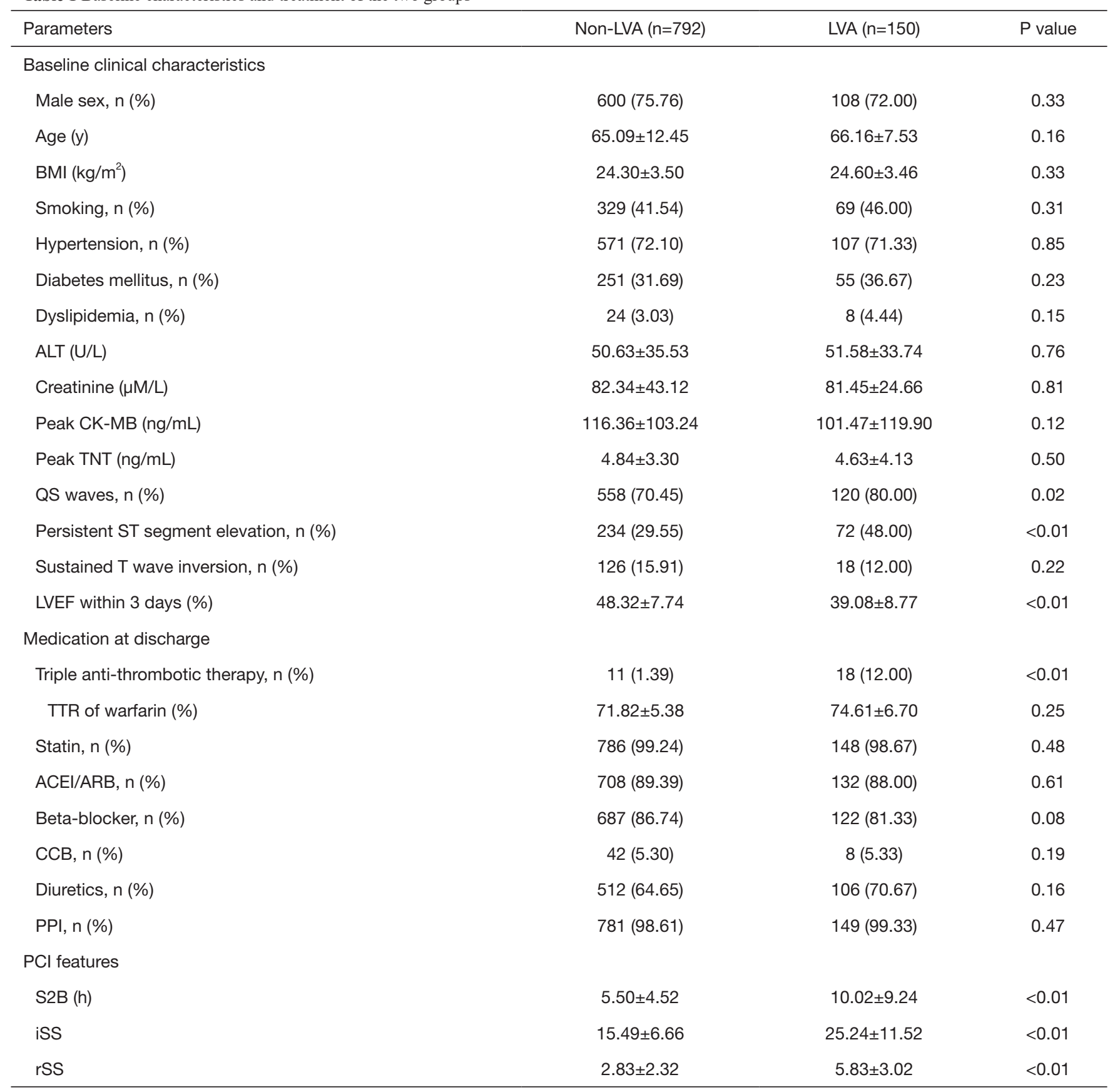

BMI, body mass index; ALT, alanine transaminase; CK-MB, creatinine kinase MB; TNT, troponin T; LVEF, left ventricular ejection fraction; $T T R$, time in therapeutic range; $\mathrm{ACEl}$, angiotensin converting enzyme inhibitor; ARB, angiotensin receptor blocker; CCB, calcium channel blocker; PPI, proton pump inhibitor; S2B, symptom-to-balloon time; iSS, initial SYNTAX Score; rSS, residual SYNTAX Score.

infarction. However, most of the previous reports were based on the entire myocardial infarction population (16-19). To avoid biases, this study focused on LVA formation due to acute anterior myocardial infarction, the data of which are limited in the contemporary PCI era. The incidence of LVA in patients with AMI was reported in the range of $5 \%$ to $15 \%$ (3). We found that $15.92 \%$ acute anterior myocardial infarction patients suffered from LVA 
Table 2 One-year clinical follow-up results

\begin{tabular}{lccc}
\hline Variables & Non-LVA, n (\%) & LVA, n (\%) & P value \\
\hline MACCEs & $51(6.44)$ & $23(15.33)$ & $12(8.00)$ \\
Cardiac death & $22(2.78)$ & $8(5.33)$ & $<0.01$ \\
TVR & $18(2.27)$ & $6(4.00)$ & 0.03 \\
Ischemic stroke & $11(1.39)$ & $24(16.00)$ & 0.03 \\
Cardiogenic shock & $24(3.03)$ & $12(8.00)$ & $<0.01$ \\
Cardiac rupture & $6(0.76)$ & $14(9.33)$ & $10(6.67)$ \\
Ventricular thrombus & $11(1.39)$ & $<0.01$ & $<0.01$ \\
Major bleeding & $19(2.40)$ & $<0.01$ \\
\hline
\end{tabular}

MACCEs, major adverse cardio-cerebral events; TVR, target vessel revascularization; LVA, left ventricular aneurysm.
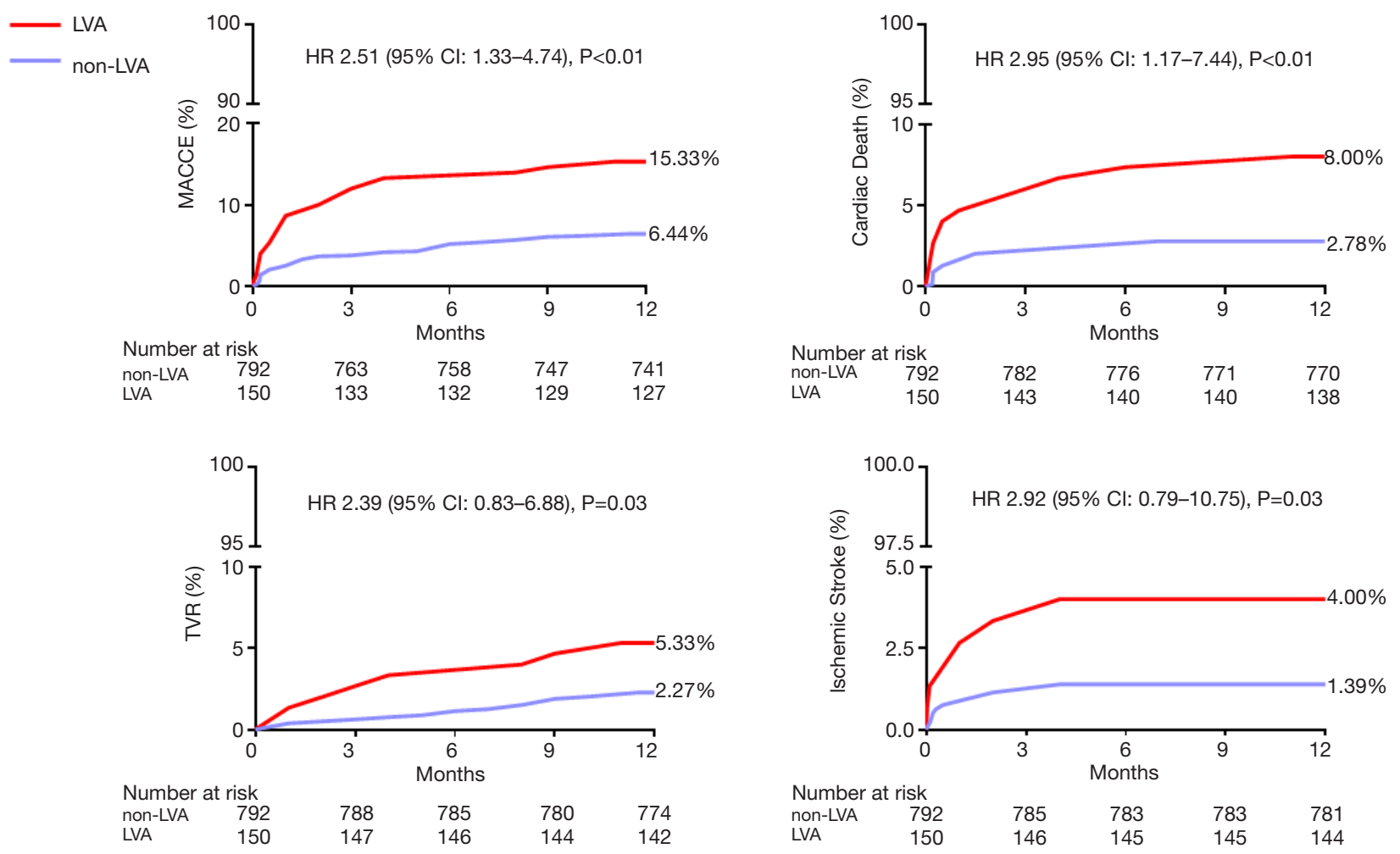

Figure 2 Adverse events of MACCEs. MACCEs, major adverse cardio-cerebral events; TVR, target vessel revascularization; LVA, left ventricular aneurysm.

after primary PCI. The incidence was slightly higher than that in previous reports because we focused on patients with acute anterior myocardial infarction, which is the high-risk subset for LVA. Due to more available and less invasive, echocardiography was adopted as the primary diagnosis of LVA in the current study, which may also elicit a higher incidence of LVA. Multivariate analysis indicated that longer S2B, increased iSS and rSS, impaired LVEF, and persistent ST segment elevation were independently associated with the occurrence of LVA. The novel findings of iSS and rSS 


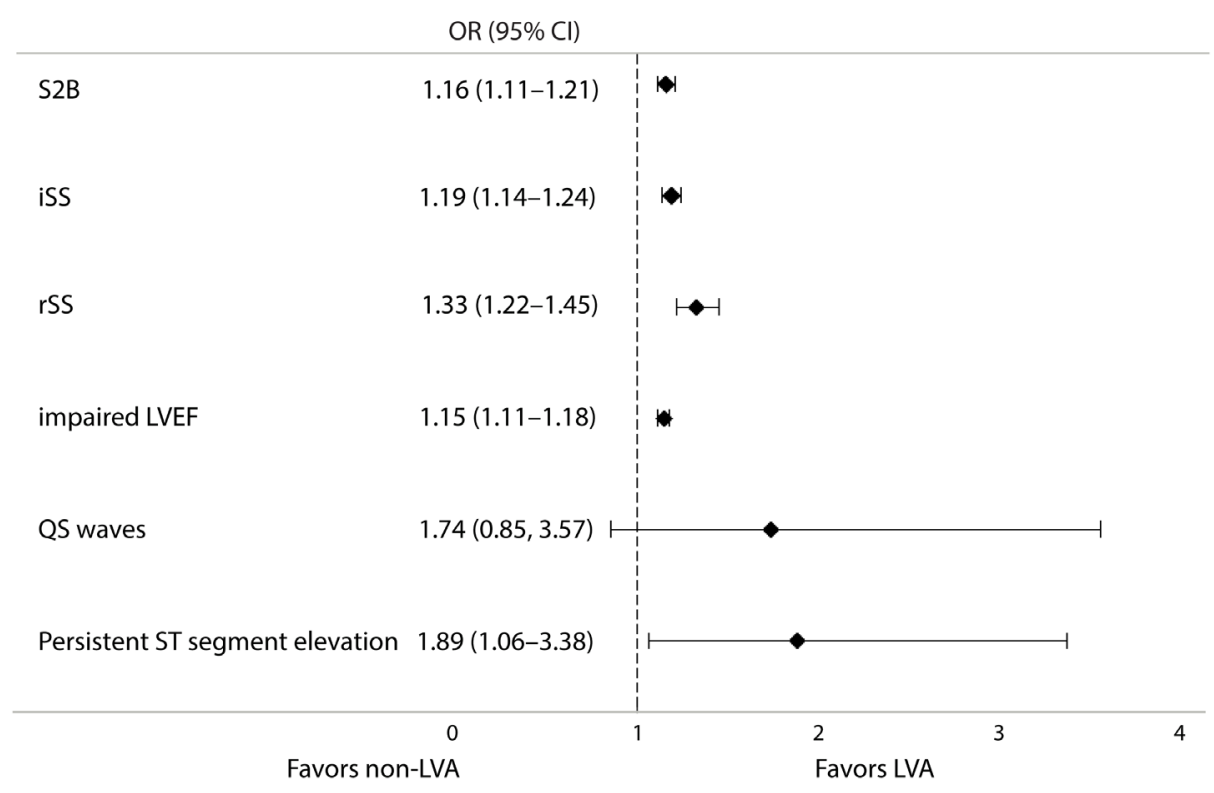

Figure 3 Forest plot for the multivariate logistical regression analysis. S2B, symptom-to-balloon time; LVEF, left ventricular ejection fraction; iSS, initial SYNTAX score; rSS, residual SYNTAX score.

as predictors of LVA may promote earlier identification of LVA. The Syntax score is easy to obtain during primary PCI, which helps clinicians evaluate the risk of LVA at an early stage. Complex coronary artery disease was an independent determinant of LVA formation. Predictors for LVA such as total occlusion of the coronary artery, worse collateral supply and incomplete revascularization demonstrated in previous studies $(6,20)$, could all be included in the Syntax score. Our results first indicated that iSS and rSS independently predicted the occurrence of LVA with optimal cutoff values of 18.75 (AUC $=0.80$, 95\% CI, 0.77-0.84, $\mathrm{P}<0.01$ ) and 3.75 (AUC $=0.82,95 \% \mathrm{CI}$, $0.79-0.85, \mathrm{P}<0.01$ ), respectively. We further demonstrated that combining the Syntax score and S2B together could more accurately predict the occurrence of LVA (iSS + S2B: $\mathrm{AUC}=0.85,95 \%$ CI, 0.82-0.88, $\mathrm{P}<0.01 ; \mathrm{rSS}+\mathrm{S} 2 \mathrm{~B}$ : AUC $=0.84,95 \%$ CI, 0.81-0.87, $\mathrm{P}<0.01)$. For patients with acute anterior myocardial infarction, these predictors may help the clinicians evaluate the probability of LVA formation and identify high-risk patients, so as to prevent LVA. It was inferred that complete and timely revascularization might help against LVA formation after acute anterior myocardial infarction.

Previous studies considered EKG as a screening tool to detect LVA. For example, persistent ST segment elevation, sustained $\mathrm{T}$ wave inversion or QS waves were found to represent the presence of LVA $(17,21)$. However, we found that in the high-risk subset of acute anterior myocardial infarction, it was difficult to identify LVA by EKG except for persistent ST segment elevation.

\section{Prognosis of LVA in the contemporary PCI era}

It has been demonstrated that LVA correlates with lifethreatening complications such as systemic embolism, ventricular arrhythmia, mechanical complications, cardiac arrest, pump failure, cardiogenic shock, ventricular thrombus, and stroke $(1,19)$. Vallabhajosyula et al. (19) verified that LVA was associated with more frequent complications, longer length of hospital stays, higher hospitalization costs, and fewer discharges to home, but without any differences in in-hospital mortality compared with the non-LVA group. The reason for comparable inhospital mortality in the LVA and non-LVA cohort in this retrospective study was at least partly due to the low incidence of primary PCI (LVA vs. non-LVA $=35.1 \%$ vs. $28.0 \%$ ) (19). So far, data on the long-term prognosis of LVA in acute anterior myocardial infarction patients treated with primary PCI remains in paucity. In the current study, the rate of MACCEs was significantly increased in the LVA group during the 1-year follow-up, which was mainly attributable to the higher occurrence of cardiac death, TVR 

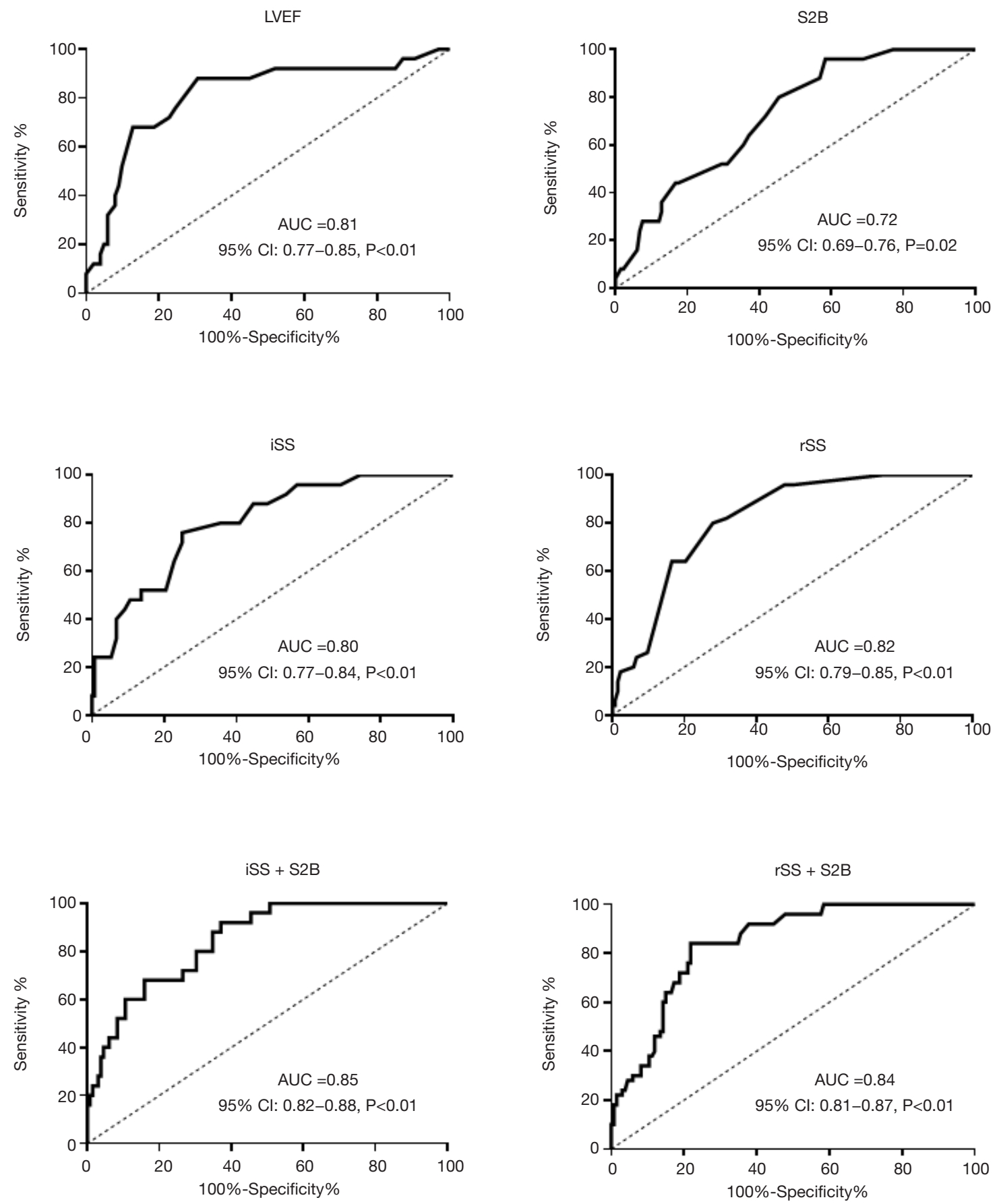

Figure 4 Receiver operating characteristic (ROC) curves. S2B, symptom-to-balloon time; iSS, initial SYNTAX score; rSS, residual SYNTAX score; LVEF, left ventricular ejection function.

and ischemic stroke. The incidence of cardiogenic shock, cardiac rupture, ventricular thrombus, and major bleeding also increased in the LVA group. These data indicated that
LVA remains a serious sequela of acute anterior myocardial infarction related to morbidity and mortality in the contemporary PCI era. 
Table 3 Predictive values of LVEF, S2B, iSS and rSS

\begin{tabular}{|c|c|c|c|c|c|c|c|}
\hline Parameters & Cutoff value & Sensitivity, \% & Specificity, \% & PPV, \% & NPV, \% & LR+ & LR- \\
\hline S2B (h) & 3.25 & 96.00 & 41.67 & 23.76 & 98.21 & 1.65 & 0.10 \\
\hline iss & 18.75 & 76.00 & 75.00 & 36.54 & 94.29 & 3.04 & 0.32 \\
\hline rSS & 3.75 & 80.00 & 72.10 & 35.19 & 95.01 & 2.87 & 0.28 \\
\hline
\end{tabular}

PPV, positive predictive value; NPV, negative predictive value; LR+, positive likelihood ratio; LR-, negative likelihood ratio; LVEF, left ventricular ejection function; S2B, symptom-to-balloon time; iSS, initial SYNTAX Score; rSS, residual SYNTAX Score.

In the current study, we found that the incidence of ventricular thrombus in the LVA group was $9.33 \%$. The result was consistent with our previous study (22). LVArelated thrombi may require long-term anticoagulation therapy which increases the hemorrhagic risk, especially in combination with DAPT after PCI (2). The proper prevention and treatment for ventricular thrombus in such populations remain ambiguous. Current guidelines recommend triple anti-thrombotic therapy (DAPT and warfarin) for up to 6 months (23). However, the INR remains controversial. Despite lower INR target of 1.5-2.0 recommended for triple anti-thrombotic therapy by previous studies (24), the risk of hemorrhagic complication was still higher in the LVA group in the current study $(6.67 \%$ vs. $2.40 \%, \mathrm{P}<0.01)$. Further randomized controlled trials about antithrombotic therapy strategy including novel oral anticoagulants are required to balance the risk of embolism and bleeding.

In conclusion, LVA is related to deteriorative clinical outcomes. For patients with acute anterior myocardial infarction undergoing primary PCI, impaired LVEF $(<40.50 \%)$, longer S2B $(>3.25 \mathrm{~h})$, increased iSS $(>18.75)$ and rSS (>3.75), and persistent ST segment elevation were independently correlated with the occurrence of LVA. These findings may help identify the LVA-prone subgroup and prevent LVA at an early stage.

\section{Limitations}

The current study had some limitations. First, the primary diagnosis of LVA was based on the ultrasonographic examination, which is not the "gold standard" for LVA detection. However, this method is widely used in clinical practice because it is more available and noninvasive. It is hypothesized that cardiac magnetic resonance imaging or nuclear imaging should be used for LVA evaluation in future studies. Second, this was a single-center analysis.
Multicenter randomized controlled trials are expected in future work.

\section{Acknowledgments}

Funding: This work was supported by the Health Science and Technology Project of Shanghai Pudong New Area Health Commission (PW2019A-13), the National Natural Science Foundation of China (81500191, 81870202, 81670228), Key Disciplines Group Construction Project of Pudong Health Bureau of Shanghai (PWZxq2017-05), and Top-level Clinical Discipline Project of Shanghai Pudong District (PWYgf2018-02).

\section{Footnote}

Reporting Checklist: The authors have completed the STARD reporting checklist. Available at http://dx.doi.org/10.21037/ jtd-20-3350

Data Sharing Statement: Available at http://dx.doi. org/10.21037/jtd-20-3350

Peer Review File: Available at http://dx.doi.org/10.21037/jtd20-3350

Conflicts of Interest: All authors have completed the ICMJE uniform disclosure form (available at http:// dx.doi.org/10.21037/jtd-20-3350). All the authors report grants from the Health Science and Technology Project of Shanghai Pudong New Area Health Commission (PW2019A-13), grants from the National Natural Science Foundation of China (81500191, 81870202, 81670228), grants from Key Disciplines Group Construction Project of Pudong Health Bureau of Shanghai (PWZxq2017-05), grants from Top-level Clinical Discipline Project of Shanghai Pudong District (PWYgf2018-02), during the 
conduct of the study.

Ethical Statement: The authors are accountable for all aspects of the work in ensuring that questions related to the accuracy or integrity of any part of the work are appropriately investigated and resolved. This study was approved by the Institutional Review Board of Shanghai East Hospital (No. 2020-096) and conducted following the principles of the Declaration of Helsinki (as revised in 2013). The written informed consent was obtained from all the patients.

Open Access Statement: This is an Open Access article distributed in accordance with the Creative Commons Attribution-NonCommercial-NoDerivs 4.0 International License (CC BY-NC-ND 4.0), which permits the noncommercial replication and distribution of the article with the strict proviso that no changes or edits are made and the original work is properly cited (including links to both the formal publication through the relevant DOI and the license). See: https://creativecommons.org/licenses/by-nc-nd/4.0/.

\section{References}

1. Ruzza A, Czer LSC, Arabia F, et al. Left Ventricular Reconstruction for Postinfarction Left Ventricular Aneurysm: Review of Surgical Techniques. Tex Heart Inst J 2017;44:326-35.

2. Hansen ML, Sorensen R, Clausen MT, et al. Risk of bleeding with single, dual, or triple therapy with warfarin, aspirin, and clopidogrel in patients with atrial fibrillation. Arch Intern Med 2010;170:1433-41.

3. Ning X, Ye X, Si Y, et al. Prevalence and prognosis of ventricular tachycardia/ventricular fibrillation in patients with post-infarction left ventricular aneurysm: Analysis of 575 cases. J Electrocardiol 2018;51:742-6.

4. Tikiz H, Balbay Y, Atak R, et al. The effect of thrombolytic therapy on left ventricular aneurysm formation in acute myocardial infarction: relationship to successful reperfusion and vessel patency. Clin Cardiol 2001;24:656-62.

5. Tikiz H, Atak R, Balbay Y, et al. Left ventricular aneurysm formation after anterior myocardial infarction: clinical and angiographic determinants in 809 patients. Int J Cardiol 2002;82:7-14; discussion 14-6.

6. Shen WF, Tribouilloy C, Mirode A, et al. Left ventricular aneurysm and prognosis in patients with first acute transmural anterior myocardial infarction and isolated left anterior descending artery disease. Eur Heart J
1992;13:39-44.

7. Feng $\mathrm{Y}$, Wang $\mathrm{Q}$, Chen $\mathrm{G}$, et al. Impaired renal function and abnormal level of ferritin are independent risk factors of left ventricular aneurysm after acute myocardial infarction: A hospital-based case-control study. Medicine (Baltimore) 2018;97:e12109.

8. Thygesen K, Alpert JS, Jaffe AS, et al. Fourth universal definition of myocardial infarction (2018). Eur Heart J 2019;40:237-69.

9. Neumann FJ, Sousa-Uva M, Ahlsson A, et al. 2018 ESC/ EACTS Guidelines on myocardial revascularization. Eur Heart J 2019;40:87-165.

10. Levine GN, Bates ER, Blankenship JC, et al. 2015 ACC/ AHA/SCAI Focused Update on Primary Percutaneous Coronary Intervention for Patients With ST-Elevation Myocardial Infarction: An Update of the 2011 ACCF/ AHA/SCAI Guideline for Percutaneous Coronary Intervention and the $2013 \mathrm{ACCF} / \mathrm{AHA}$ Guideline for the Management of ST-Elevation Myocardial Infarction: A Report of the American College of Cardiology/American Heart Association Task Force on Clinical Practice Guidelines and the Society for Cardiovascular Angiography and Interventions. Circulation 2016;133:1135-47.

11. Zhang Q, Wang XL, Liao ML, et al. Periprocedural use of tirofiban in elective percutaneous coronary intervention for long coronary lesions in stable patients with overlapping drug-eluting stents--the PETITION study: a prospective, randomized, multicenter study. Catheter Cardiovasc Interv 2015;85 Suppl 1:762-9.

12. van Diepen S, Katz JN, Albert NM, et al. Contemporary Management of Cardiogenic Shock: A Scientific Statement From the American Heart Association. Circulation 2017;136:e232-e268.

13. Jentzer JC, van Diepen S, Barsness GW, et al. Cardiogenic Shock Classification to Predict Mortality in the Cardiac Intensive Care Unit. J Am Coll Cardiol 2019;74:2117-28.

14. Kernan WN, Ovbiagele B, Black HR, et al. Guidelines for the prevention of stroke in patients with stroke and transient ischemic attack: a guideline for healthcare professionals from the American Heart Association/ American Stroke Association. Stroke 2014;45:2160-236.

15. Mehran R, Rao SV, Bhatt DL, et al. Standardized bleeding definitions for cardiovascular clinical trials: a consensus report from the Bleeding Academic Research Consortium. Circulation 2011;123:2736-47.

16. Pang J, Zhang Z, Zheng TZ, et al. The analysis of related factors of ventricular aneurysm formation in patients with acute myocardial infarction in northwest of China. Int $\mathrm{J}$ 
Cardiol 2015;181:50-2.

17. Zhang Z, Guo J. Predictive risk factors of early onset left ventricular aneurysm formation in patients with acute STelevation myocardial infarction. Heart Lung 2020;49:80-5.

18. Celebi S, Celebi OO, Cetin S, et al. The Usefulness of Admission Plasma NT-pro BNP Level to Predict Left Ventricular Aneurysm Formation after Acute ST-Segment Elevation Myocardial Infarction. Arq Bras Cardiol 2019;113:1129-37.

19. Vallabhajosyula S, Kanwar S, Aung H, et al. Temporal Trends and Outcomes of Left Ventricular Aneurysm After Acute Myocardial Infarction. Am J Cardiol 2020;133:32-8.

20. Mori M, Sakakura K, Wada H, et al. Left ventricular apical aneurysm following primary percutaneous coronary intervention. Heart Vessels 2013;28:677-83.

21. Marzlin KM. Ventricular Aneurysm: Diagnosis and

Cite this article as: You J, Gao L, Shen Y, Guo W, Wang X, Wan Q, Wang X, Wu J, Zhang Q. Predictors and longterm prognosis of left ventricular aneurysm in patients with acute anterior myocardial infarction treated with primary percutaneous coronary intervention in the contemporary era. J Thorac Dis 2021;13(3):1706-1716. doi: 10.21037/jtd-20-3350
Treatment. AACN Adv Crit Care 2017;28:391-4.

22. You J, Wang X, Wu J, et al. Predictors and prognosis of left ventricular thrombus in post-myocardial infarction patients with left ventricular dysfunction after percutaneous coronary intervention. J Thorac Dis 2018;10:4912-22.

23. McCarthy CP, Vaduganathan M, McCarthy KJ, et al. Left Ventricular Thrombus After Acute Myocardial Infarction: Screening, Prevention, and Treatment. JAMA Cardiol 2018;3:642-9.

24. You J, Li H, Guo W, et al. Platelet function testing guided antiplatelet therapy reduces cardiovascular events in Chinese patients with ST-segment elevation myocardial infarction undergoing percutaneous coronary intervention: The PATROL study. Catheter Cardiovasc Interv 2020;95 Suppl 1:598-605. 